\title{
Molecular mechanism for the regulation of yeast separase by securin
}

\author{
Shukun Luo \& Liang Tong \\ Department of Biological Sciences, Columbia University, New York, New York 10027, USA
}

Cell division is a crucial event for the continuation of life. During this process, one of the pivotal steps is the proper segregation of the duplicated chromosomes, which are held together by the cohesin ring. Separase is a cysteine protease that opens the cohesion ring by cleaving its kleisin subunit at the early stage of anaphase. In human tumors separase is overexpressed, making it a potential target for drug discovery. The protease activity of separase is strictly regulated by the inhibitor securin, which forms a tight complex with separase and may also stabilize this enzyme. Separases are large, 140-250 kDa enzymes, with an N-terminal $\alpha$-helical region and a C-terminal caspase-like catalytic domain. While the function of separase has been widely studied, the atomic structures of full-length separase and especially its complex with securin remain unknown.

We have determined the crystal structures of the yeast Saccharomyces cerevisiae separasesecurin complex at up to $2.6 \AA$ resolution. The $\alpha$-helical region of separase (also known as Esp1) contains four domains (I-IV), and a substrate-binding domain immediately precedes the catalytic domain and has tight associations with it. The separase-securin complex assumes a highly elongated structure. Residues 258-373 of securin (Pds1), named the separase interaction segment, are primarily in an extended conformation and traverse the entire length of separase, interacting with all of its domains. Most importantly, residues 258-269 of securin are located in the separase active site, illuminating the mechanism of inhibition. Biochemical studies confirm the structural observations and indicate that contacts outside the separase active site are crucial for stabilizing the complex, thereby defining an important function for the $\alpha$-helical region of separase. Together, our findings shed light on the molecular mechanism of separase and its inhibition by securin.

Reference:

Luo, S. \& Tong, L. Molecular mechanism for the regulation of yeast separase by securin. Nature 542, 255-259, doi:10.1038/nature21061 (2017). 